\title{
Ein Kassenmodell für mündige Patienten
}

\section{Urs Strebel}

Dr. med., Facharzt für Innere Medizin, Hämatologie und Onkologie, ehem. Chefarzt für Innere Medizin am Spital Männedorf, Mitglied FMH

Nach 40-jähriger klinischer Tätigkeit - vorwiegend im Spital, aber auch zwei Jahre als selbständiger Hausarzt - und nach dem Rücktritt von verschiedenen Aufgaben bei der FMH, SGIM und SAMW per Ende 2015 hatte ich mir vorgenommen, die anstehenden Fragen im Gesundheitswesen jüngeren Mitbürgerinnen und Mitbürgern ${ }^{1}$ zur Lösung zu überlassen. Trotzdem verfolge ich die gesundheitspolitischen Themen weiter. Dabei beschäftigt mich auch das Kostenproblem, das u.a. durch falsche Anreize, Überbehandlungen, steigende Medikamentenpreise und Pseudowettbewerb bedingt ist (siehe auch [1-3]). Ich fühle mich deshalb genötigt, auf zwei Massnahmen hinzuweisen, die das System verbessern könnten.

\section{Schaffung einer Qualitätskontrolle der Indikation}

Die erste Massnahme läge in der Einführung einer Qualitätskontrolle der Indikation. Heute werden nur einzelne Schritte in der Behandlungskette und/oder das Resultat auf ihre Qualität hin kontrolliert, nicht aber die Indikation. Man denke an die unterschiedlichen Kaiserschnittraten, die verschiedenen Behandlungen der vorderen Kreuzbandruptur oder die Indikation zur Vertebroplastie [4]. Im Club vom 22. November 2016 hat auch der Präsident der fmCh diese fehlende Qualitätskontrolle bemängelt. Und der im ZV der FMH für das Ressort Qualität zuständige Vertreter hat mir seit 2012 mehrfach erklärt, dass eine Qualitätskontrolle der Indikation nötig und deren Einführung geplant sei, allerdings nicht mit oberster Priorität. Da bin ich anderer Meinung. Denn wegen ihrer zentralen Bedeutung sollte sie möglichst rasch realisiert werden.

\section{Einführung eines unbegrenzten Selbst- behalts}

Die zweite Massnahme betrifft Franchise und Selbstbehalt. Aktuell müssen die ersten 300 Franken pro Kalenderjahr selber bezahlt werden. Von den die Franchise übersteigenden Kosten müssen dann bis maximal 700 Franken pro Jahr, zehn Prozent, als Selbstbehalt entrichtet werden [5].

Franchise und Selbstbehalt haben sicher einen bremsenden Einfluss auf die ersten Arztbesuche. Sobald ein Patient aber die 1000 Franken bezahlt hat, entfällt diese Bremse. Denn alle weiteren Leistungen im gleichen Kalenderjahr sind von nun an gratis. Eine Folge

Ich halte ein System ohne Franchise, aber mit unbegrenztem Selbstbehalt für geeigneter.

davon ist, dass wir Ärzte nie über Kosten sprechen müssen und die Patienten Kostenargumente nur schwer akzeptieren und meist mit dem Hinweis zurückweisen, dass sie schliesslich hohe Prämien bezahlen. Eine direkte Folge ist die Zunahme von Wahleingriffen in der zweiten Jahreshälfte, wenn viele Patienten, die Anfang Jahr Franchise und Selbstbehalt bereits bezahlt haben, den Wahleingriff noch im gleichen Kalenderjahr kostenfrei durchführen lassen möchten.

Ich halte ein System ohne Franchise, aber mit unbegrenztem Selbstbehalt für geeigneter: Patienten würden so eher von unüberlegten Arztbesuchen und von der Einforderung sinnloser Zusatzuntersuchungen und Therapien abgehalten und wir Ärzte von unnötigen Abklärungen, überteuerten Therapien und Eingriffen, deren Indikation diskutabel ist. Denn wenn der Pa- 


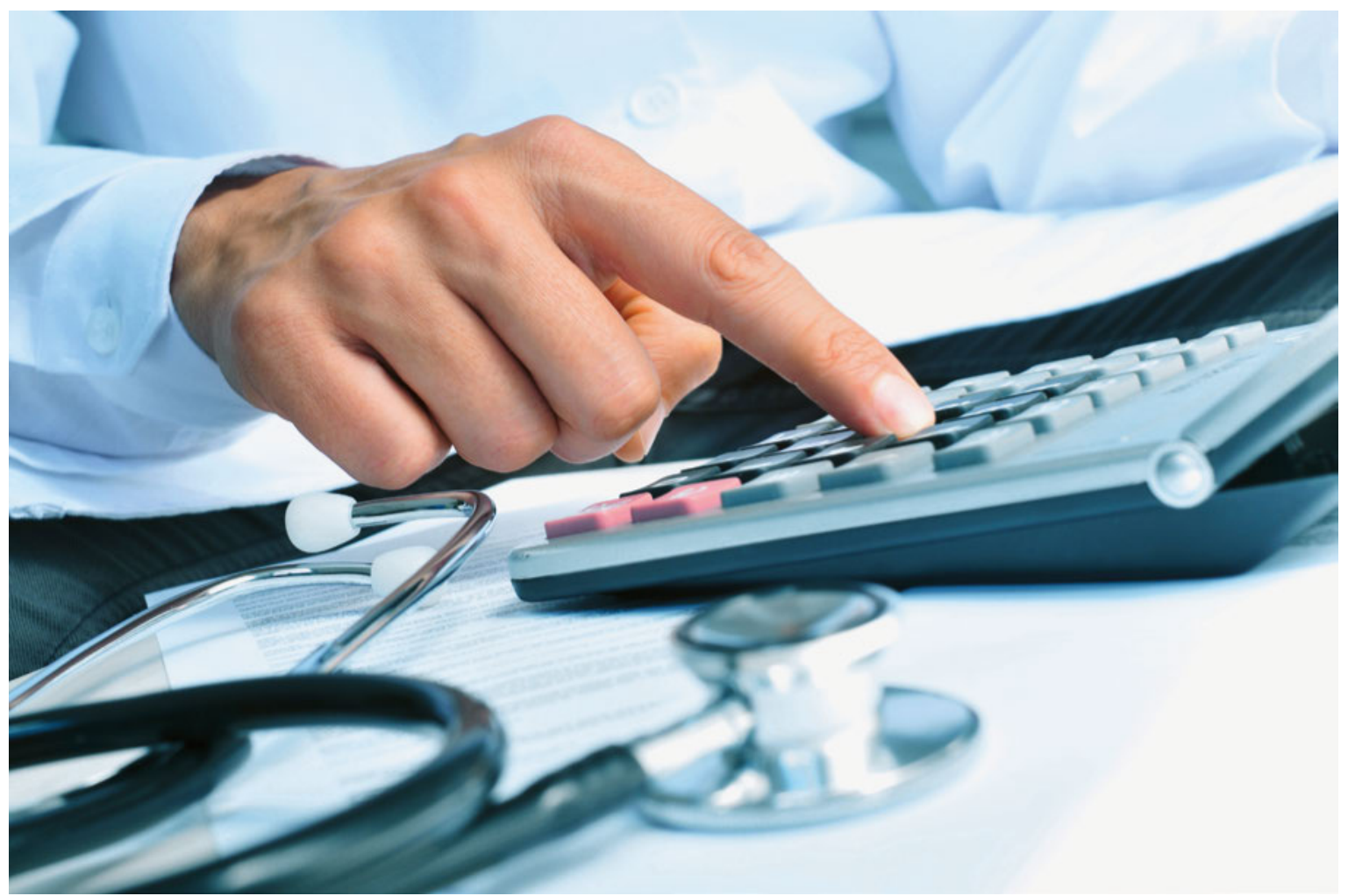

Die Kosten im Gesundheitswesen steigen: Ein Kassenmodell, in dem die Indikation kontrolliert und ein unbegrenzter Selbstbehalt gelten würde, könnte Abhilfe schaffen.

tient stets einen Teil mitbezahlen muss, sind wir gezwungen, über die Kosten zu reden und zu begründen, weshalb die kostspieligere Massnahme besser ist als die günstigere. Ich bin auch sicher, dass unter diesen Umständen nicht alle Patienten all das möchten, was an teuren Therapien mit lediglich geringem $\mathrm{Zu}$ satznutzen angeboten wird. Damit würde das Gesundheitswesen endlich in eine Wettbewerbssituation geraten, in welcher der Preis auch von der Nachfrage mitbestimmt wird (siehe Bemerkung 1).

\section{Überlegungen zu Modellen über den Selbstbehalt}

Ich habe meine Überlegungen in den letzten Monaten mit einigen mir bekannten Parlamentariern von FDP, CVP und SP besprochen. Von FDP und CVP kamen wohlwollende Rückmeldungen. Allerdings erklärte sich niemand bereit, das Thema im Rat im Rahmen der Debatte über die Erhöhung der Franchise aufzunehmen. Das grösste Interesse zeigte eine Nationalrätin der SP. Sie gab allerdings offen zu, dass es nicht möglich sein dürfte, ihre Parteigenossen von der Idee eines unlimitierten Selbstbehaltes zu überzeugen, da sie tiefere Einkommensklassen benachteiligen und unbemittelte Menschen dazu verleiten könnte, im Notfall aus Kostengründen auf medizinische Hilfe zu verzichten. Einen weiteren Einwand stellt die auf den ersten Blick berechtigte Angst der Benachteiligung von Patienten dar, die sehr teure Massnahmen benötigen, sowie von Patienten mit chronischen und/oder seltenen Krankheiten.

Ein unbegrenzter Selbstbehalt braucht deshalb gute flankierende Massnahmen, denn er darf nicht zur Folge haben, dass notwendige Therapien nicht durchgeführt werden können. Was den Prozentsatz des Selbstbehalts betrifft, kann man sich neben einem linearen Satz von beispielsweise $10 \%$ auch einen degressiven von $12 \%$ für die ersten 10000 Franken, 9\% für die nächsten 1000 Franken und 6\% für die weiteren Kosten innerhalb von 12 Monaten vorstellen. Armen Patienten mit einer chronischen Krankheit müssten die Kosten für diese Krankheit eventuell ganz erlassen

\section{Falls sich die Patienten an den Kosten} beteiligen müssten, würden sie ihre eigenen Kosten-Nutzen-Überlegungen anstellen.

werden. Natürlich müsste das Modell von Fachleuten durchgerechnet und angepasst werden, die mehr von Ökonomie verstehen und die Gesundheitsdaten besser kennen als ich.

Ich möchte noch einige Überlegungen zu den beiden Modellen anstellen: 
- Ein Vorteil des bisherigen Modells ist, dass sich alle in der Schweiz wohnhaften Personen obligatorisch versichern müssen. Das muss so bleiben.

- Beim aktuellen Modell müssen die ersten 300 Franken aus der eigenen Tasche bezahlt werden. Für Wenig-Verdienende ist das eine nicht zu unterschätzende Hürde.

- Franchise und Selbstbehalt gelten pro Kalenderjahr. In einem neuen Modell würde bei einer linearen Beteiligung eine zeitliche Limite entfallen, bei einem degressiven Modell würde sie 12 Monate betragen.

- Sobald die Kasse leistungspflichtig wird, vergütet sie dafür alle innerhalb des Kalenderjahres auftretenden Krankheiten, auch wenn diese in keiner Weise zusammenhängen wie etwa Diskushernie und Gallenkolik. Während das im bisherigen Modell kostentreibend wirkt, spielt es bei einer unlimitierten Selbstbeteiligung keine Rolle.

- Rund 30\% der Einwohner erhalten Subventionen. Diese flankierende Massnahme bräuchte es in angepasster Form auch im neuen Modell.

- Bei einer unbegrenzten Selbstbeteiligung entfällt die Franchise als anfängliche Hürde. Denn 10\% einer einfachen Konsultation entsprechen 10 bis 30 Franken und nur in den seltensten Fällen 300 Franken oder mehr. Es besteht deshalb gerade für WenigVerdienende keine hohe Anfangshürde.

Abschliessend möchte ich drei Bemerkungen anfügen und meine Kernanliegen wiederholen:

Bemerkung 1: Einzelne Medikamente kosten mehrere 10000 Franken pro Jahr, wobei ihr Nutzen teilweise bescheiden ist [6]. Die Preisgestaltung lässt sich nicht transparent nachvollziehen. Aufgrund der Dividenden und Boni darf aber davon ausgegangen werden, dass die Pharmaindustrie den Preis einiges über den Gestehungskosten ansetzt. Sie senkt ihn auch nicht freiwillig, wenn die Indikation breiter und das Medikament häufiger verkauft wird. Da die Patienten wegen ihrer Grundkrankheit Franchise und Selbstbehalt in der Regel schon bezahlt haben, kommt von ihrer Seite kein Druck, den Preis zu senken. Und auch das BAG hat nur einen beschränkten Einfluss. Falls sich die Patienten an den Kosten beteiligen müssten, würden sie ihre eigenen Kosten-Nutzen-Überlegungen anstellen und zum Beispiel bei fortgeschrittenem Alter teilweise auf eine teure Therapie verzichten. Unter diesen Bedingungen wird sich die Pharmaindustrie überlegen, ob sie nicht den Preis senken will, um dafür mehr zu verkaufen.

Bemerkung 2: Zur Diskussion über ein neues Kassenmodell gehören auch die Fragen, ob Kopfprämien gerecht sind und ob die Unterteilung in ambulante und stationäre Versorgung mit unterschiedlichen Kostenträgern noch zeitgemäss ist.

Bemerkung 3: Wenn wir über die Kosten des Gesundheitswesens und mögliche Verbesserungen nachdenken, dürfen wir uns nicht auf die Kassenprobleme beschränken, sondern müssen auch die Tatsache berücksichtigen, dass sozio-ökonomisch benachteiligte Menschen eine signifikant verkürzte Lebenserwartung haben [7, 8]. Überlegungen, wie diese Situation zu verbessern ist, gehören auch zu einem umfassenden Gesundheitswesen.

Um die Diskussion noch einmal $\mathrm{zu}$ fokussieren, möchte ich die beiden zentralen Gedanken wiederholen.

- Einführung einer Qualitätskontrolle der Indikationen;

- unbegrenzter Selbstbehalt ohne Franchise.

Ohne diese oder ähnliche Massnahmen droht uns eine Rationierung, die sicher niemand will.

\section{Danksagung}

Ich danke Prof. Dr. Markus Zimmermann und Irmgard Strebel herzlich für die kritische und konstruktive Durchsicht des Manuskripts.

\section{Literatur}

1 Teuwsen P. \& Mijuk G. Ist das wirklich alles nötig? NZZ am Sonntag 30.10.16, S. $22 \mathrm{f}$

2 Scheidegger D. Ärzte verhalten sich wie Messies. Tages-Anzeiger 23.11.16, S. 40.

3 Oertle D. Die Hausärzte sterben aus: Na und? In: Schweiz Ärztezeitung 2016;97(51-52):1804-180.

4 http://www.medical-board.ch/index.php?id=809\&L=1\%27A\%3D0

5 http://www.bag.admin.ch/themen/krankenversicherung/04114/0 4285/?lang=de

6 Schrag D. The Price Tag on Progress - Chemotherapy for Colorectal Cancer. NEJM 2004:317:317-9.

7 http://www.humanrights.ch/de/menschenrechte-schweiz/ inneres/bildung/sozialrechte/einfluss-soziooekonomischerfaktoren-gesundheit

8 http://www.lebenserwartung.info/index-Dateien/soziodiff.htm

\section{Bildnachweis}

(c) Nito100 | Dreamstime.com 